Website: http://revistas.lamolina.edu.pe/index.php/acu/index

(C) Universidad Nacional Agraria La Molina, Lima - Perú

\title{
Impacto del Acuerdo de Promoción Comercial entre el Perú y los Estados Unidos en la demanda de maíz amarillo duro
}

\author{
Impact of the Trade Promotion Agreement between Peru and the United States on the Corn \\ Demand
}

\author{
Humberto Alejandro Trujillo Cubillas ${ }^{1}$
}

\section{Resumen}

El objetivo del presente estudio fue evaluar el impacto del Acuerdo de Promoción Comercial (APC) entre el Perú y los Estados Unidos en la demanda de Maíz Amarillo Duro (MAD) en el Perú. Esto permitió responder diferentes pronósticos que se hicieron sobre el impacto del APC en el sector de MAD. En este estudio se recurrió a estadísticas de comercio exterior, del sector agricultura y económicas y para los determinantes de la demanda de MAD, se utilizó una regresión econométrica.

Palabras clave: APC; Maíz; Comercio Exterior; Demanda.

\begin{abstract}
The objective of the present study was to evaluate the Trade Promotion Agreement TPA between Peru and the United States on the demand for Corn in Peru. This allowed us to answer different forecasts that were made about the impact of the TPA in the Corn sector. In this study, we used statistics on foreign trade, the agriculture and economic sectors, and for the determinants of Corn demand, an econometric regression was used.
\end{abstract}

Keywords: TPA; Corn; International Trade; Demand.

\section{Introducción}

El APC entre Estados Unidos y el Perú, es más conocido como Tratado de Libre Comercio (TLC), entró en vigencia fen ebrero de 2009. Las estimaciones para el sector de Maíz Amarillo Duro (MAD) eran adversas para algunos, dado los resultados observados en otros países, mientras que para otros no lo sería. Estos pronósticos se basaban en que el APC implicaba un sistema de cuotas incremental para la apertura de las importaciones de MAD desde Estados Unidos al Perú. Este esquema estaba previsto para 12 años (Tabla 1).

Tabla 1. Cuotas de importación de MAD al Perú desde EE.UU. en el marco del APC

\begin{tabular}{cc}
\hline Año & $\begin{array}{c}\text { Cantidad } \\
\text { (Toneladas Métricas) }\end{array}$ \\
\hline 1 & 500,000 \\
2 & 530,000 \\
3 & 561,800 \\
4 & 595,508 \\
5 & 631,238 \\
6 & 669,113 \\
7 & 709,260 \\
8 & 751,815 \\
9 & 796,924 \\
10 & 844,739 \\
11 & 895,424 \\
12 & Ilimitada \\
\hline Fuente: APC EE.UU. - Perú
\end{tabular}

\section{El TLCAN y en impacto en sector del maíz en}

\section{México}

En TLCAN entró en vigencia en enero de 1994 y tuvo un impacto negativo en el sector del maíz según la investigación de De Ita (2008) donde dice: La "protección extraordinaria" ha sido eliminada desde 1996 por decisión unilateral del gobierno mexicano. El incremento de las importaciones desde EE.UU. no se debió a una falta de producción en México o a precios internos mayores a los internacionales, sino al programa de apoyo a las exportaciones agropecuarias de EE.UU. Desde 1996 el sector ganadero paso a ser el principal destino de las importaciones. Los consumidores de granos evitaron que se cobraran los altos aranceles permitidos por el TLCAN. Esto afecto a los productores pequeños. La política de cupos mayores a los negociados en el TLCAN implico una mayor carga a los campesinos. En 1999 se eliminó la empresa estatal Conasupo, encargada de regular el mercado de los granos en apoyo a los productores y consumidores. Las empresas transnacionales se convirtieron en las únicas compradoras de las cosechas. El incremento de importaciones redujo el precio interno del maíz en 59\% entre 1991 y 2006. En el año 2001, el 47,1\% de las importaciones se destinó al sector pecuario, el 31,2\% al sector almidonero, y el $11 \%$ al sector harinero. En ese mismo año, la mitad de las importaciones las absorbieron nueve grandes empresas. 
Según Moreno et al. (2016) encontraron lo siguiente: El Índice de Dependencia muestra una tendencia creciente a partir de 1994. Recomienda revisar el papel que ha desempeñado el Estado en cuanto a la protección del comercio y fomento de la producción del grano en México. Plantea la necesidad de una política de fomento eficiente y efectivo.

\section{Los pronósticos en relación APC Perú-EE.UU}

Según el estudio del IICA realizado por Cafferata y Arias (2007) estimaban que el APC tendría los siguientes efectos en sector de maíz: La cadena de maíz amarillo duro aves - cerdo mantendría su competitividad en el mercado interno con respecto a las potenciales importaciones de EEUU. La compensación a favor de los productores de maíz comprometida por el gobierno peruano durante la negociación del APC debería ayudar a continuar con una tendencia creciente en los niveles de producción y rendimiento del MAD. Según documento de CEPESCONVEAGRO (2009), denominado Impacto del TLC Perú-EE.UU. en el maíz amarillo duro - Preliminar, las consecuencias del TLC serían:

"Una consecuencia negativa de la medida dispuesta es que se debilita aún más la posición comercial negociadora a nivel externo como interno. En tal sentido, a nivel externo, se reducen las herramientas arancelarias a negociar ante cualquier proceso de negociación comercial internacional (TLC CAN-UE, Canadá, México, China etc.).

A nivel interno, las medidas dispuestas no permitirán necesariamente que se reduzcan los precios de los productos dirigidos al consumidor final, debido a que, como se conoce, los mercados de comercialización agrarios se ven expuestos a la posición de dominio sea de los importadores como de los agiotistas que comercializan la producción en el interior del país y que utilizan y especulan, de acuerdo al costo de importación, el precio que pagan al agricultor en chacra.

En suma, esta estructura comercial impide que el efecto de abaratamiento de las importaciones se traduzca en menores precios al consumidor final, por lo que no se precisaría un adecuado control inflacionario con las medidas adoptadas"

Finalmente, según el informe del MINAG (2006) denominado Informe Técnico Resultados del Tratado de Libre Comercio Perú- Estados Unidos. Sector Agricultura. Las consecuencias para el sector del maíz amarillo duro serían: Las importaciones de maíz procedentes de EE.UU. se incrementarían. Asumiendo que la reducción del arancel implique una disminución en el precio nacionalizado, esto se trasladaría al precio nacional registrando la misma magnitud de disminución. La aplicación de la compensación haría que no existan diferencias entre el precio del maíz importado de EE.UU. y el MAD nacional.

"Respecto a los cambios en el valor bruto de la producción, los sectores que saldrían más perjudicado ante la entrada en vigor del Tratado de Libre Comercio con los Estados Unidos serían el maíz amarillo duro y el trigo, ya que experimentarían una reducción en el VBP como respuesta a una caída en la producción doméstica $y$ en los precios domésticos producto de un incremento marginal en las importaciones totales $(1,85 \%$ para el caso de maíz y el $2 \%$ para el caso del trigo)".

Un cuadro resumen de los impacto del APC Perú-EE. UU. se presenta en la Tabla 2.

Tabla 2. Pronósticos para el MAD en el Perú como consecuencia del APC Perú-EE.UU

\begin{tabular}{lll}
\hline \multicolumn{1}{c}{ Variable } & \multicolumn{1}{c}{ IICA } & \multicolumn{1}{c}{ MINAG } \\
\hline Importación & Se incrementará \\
Producción & $\begin{array}{l}\text { Tendencia creciente } \\
\text { con ayuda de la } \\
\text { compensación. }\end{array}$ & Caída en la producción. \\
\hline Precio & & Caída en el precio. \\
\hline
\end{tabular}

\section{Contraste con lo sucedido}

Al contrastar los pronósticos con la realidad se encuentra que si bien inicialmente se incrementó las importaciones de MAD desde los EE.UU. del año 2009 al 2010. Luego disminuyeron los años 2011 al 2013, influenciadas por la caída en la producción de MAD en los EE.UU. los años 2011 y 2012, para volver a subir desde el año 2014, pero subió a un ritmo mayor al que estuvo proyectado por la cuota de importación del APC (Tabla 3).

Tabla 3. Importaciones al Perú de MAD según origen y cuota de Importación en el APC

(en Ton. / PA: 1005.90.11.00)

\begin{tabular}{rrrrr}
\hline Año & \multicolumn{1}{c}{ EE.UU. } & Argentina & \multicolumn{1}{l}{ Total } & Cuota de M \\
\hline 2000 & 282,522 & 562,924 & 846,424 & \\
2001 & 203,883 & 614,501 & 843,970 & \\
2002 & 188,257 & 735,208 & 927,098 & \\
2003 & 65,473 & 822,368 & 966,644 & \\
2004 & 230,251 & 815,290 & 1086,892 & \\
2005 & 199,739 & 1095,325 & 1300,386 & \\
2006 & 371,281 & 977,123 & 1502,501 & \\
2007 & 407,520 & 1084,179 & 1523,121 & \\
2008 & 214,638 & 1070,635 & 1391,379 & \\
2009 & 572,060 & 692,402 & 1499,244 & 500,000 \\
2010 & 626,370 & 1059,484 & 1903,272 & 530,000 \\
2011 & 63,131 & 1514,983 & 1897,582 & 561,800 \\
2012 & 45,240 & 1242,272 & 1831,245 & 595,508 \\
2013 & 219,624 & 1377,533 & 2005,137 & 631,238 \\
2014 & 1698,511 & 571,014 & 2315,888 & 669,113 \\
2015 & 2129,767 & 345,014 & 2661,281 & 709,260 \\
2016 & 2857,079 & 142,005 & 3020,775 & 751,815 \\
\hline
\end{tabular}

El crecimiento de las importaciones desde Estados Unidos, más allá de las cuotas establecidas en el APC, no solo hizo que las importaciones de Argentina disminuyeran sino que el volumen total se incremente en forma sostenida desde el año 2012. El impacto en la producción nacional 
según el informe del Ministerio de Agricultura sería la disminución de la producción. Mientras que para el IICA la tendencia creciente de la producción continuaría debido a las medidas de compensación adoptadas. Lo que sucedió fue que la producción ha tenido una tendencia creciente en el periodo de vigencia del APC, que es desde el año 2009, a pesar de que no se implementaron las medidas de compensación para los productores (Tabla 4).

También se pronosticó que el precio interno disminuiría, pero se aprecia en la Tabla 4 que los precios en chacra han venido creciendo. Solo el año 2015 se mantuvo estable probablemente por el notable incremento en la producción registrado ese año.

Lo que nos lleva a preguntar por qué no sucedió lo que se pronosticó. Lo que parece que sucedió es que a pesar del incremento de las importaciones no aumento la dependencia por maíz importado y, unido al crecimiento de la demanda, que a su vez está influenciada por el crecimiento económico hizo que la producción crezca aun con marchas y contramarchas.

Tabla 4. Producción y precio del MAD en el Perú

\begin{tabular}{ccccc}
\hline Año & $\begin{array}{c}\text { Producción } \\
\text { (Ton.) }\end{array}$ & $\begin{array}{c}\text { Crecimiento } \\
(\%)\end{array}$ & $\begin{array}{c}\text { Crec. } \\
\text { Acum.* } \\
(\%)\end{array}$ & $\begin{array}{c}\text { Precio } \\
\text { Chacra } \\
\text { (Soles/Kg) }\end{array}$ \\
\hline 2000 & 960,362 & & & 0,51 \\
2001 & 1057,355 & $10 \%$ & & 0,51 \\
2002 & 1038,117 & $-2 \%$ & & 0,51 \\
2003 & 1097,337 & $6 \%$ & & 0,49 \\
2004 & 983,156 & $-10 \%$ & & 0,54 \\
2005 & 999,274 & $2 \%$ & & 0,49 \\
2006 & 1020,042 & $2 \%$ & & 0,53 \\
2007 & 1122,918 & $10 \%$ & & 0,66 \\
2008 & 1231,516 & $10 \%$ & & 0,74 \\
2009 & 1273,943 & $3 \%$ & & 0,68 \\
2010 & 1283,621 & $1 \%$ & $1 \%$ & 0,75 \\
2011 & 1260,123 & $-2 \%$ & $-1 \%$ & 0,91 \\
2012 & 1392,972 & $11 \%$ & $9 \%$ & 0,83 \\
2013 & 1365,239 & $-2 \%$ & $7 \%$ & 0,88 \\
2014 & 1227,562 & $-10 \%$ & $-4 \%$ & 0,90 \\
2015 & 1438,562 & $17 \%$ & $13 \%$ & 0,90 \\
\hline Fuente: Ministerio de Agricultura 02006. & &
\end{tabular}

* El crecimiento acumulado es respecto al año 2009

\section{Materiales y métodos}

Una manera de medir la dependencia por el maíz amarillo duro importado es el Índice de Dependencia (ID) desarrollado por la FAO (2010) para estimar la dependencia en cereales. El índice se obtiene al dividir el volumen de importaciones entre el resultado de sumar la producción más las importaciones menos las exportaciones. La fórmula del ID es:

ID $=$ importaciones $/$ (producción + importaciones exportaciones)

Dado que no se registran exportaciones el ID se calcularía así:
ID = importaciones $/($ producción + importaciones $)$

En base al concepto balanza disponibilidad desaparición se establece que la disponibilidad total de maíz será igual a la desaparición. A su vez la disponibilidad está dada por la producción nacional y por las importaciones, mientras que la desaparición total del maíz está dada por el consumo doméstico más las exportaciones.

$$
\mathrm{Qt}+\mathrm{Mt}=\mathrm{Dt}+\mathrm{Xt}
$$

Donde: Qt: Producción nacional de maíz en el año t

Mt: Importación de maíz en el año $t$

Dt: Demanda de maíz en el año $t$

$\mathrm{Xt}$ : Exportaciones de maíz en el año $\mathrm{t}$

Despejando la demanda se tiene que: $\mathrm{Dt}=\mathrm{Qt}+\mathrm{Mt}-\mathrm{Xt}$

Como en el Perú no se registran exportaciones de maíz la ecuación se reduce a: $\mathrm{Dt}=\mathrm{Qt}+\mathrm{Mt}$

Por otro lado la demanda está determinada por algunos factores:

$\mathrm{Dt}=\mathrm{f}(\mathrm{PD}, \mathrm{PIB}, \mathrm{POB}, \mathrm{D}, \varepsilon \mathrm{t})$

Donde: Dt: Demanda de maíz en el año $t$

PDt: Precio de demanda en el año $t$

POBt: Población en el año $t$

D: Variable Dummy con valor de 1 para el APC Perú EE.UU.

$\varepsilon t$ : Error.

Aunque en el caso del Perú la población no consume de manera directa el MAD sino que lo hace a través de los productos que lo utilizan como insumo, no ha sido posible medir la importancia relativa que cada tipo de carne tiene en el consumo de MAD, por lo que utilizará la población en el modelo al ser el receptor final de las carnes que son alimentadas con MAD.

\section{Resultados y discusión}

La demanda se estimó con a través de la agregación de la importación más la producción. Esta demanda se mantuvo creciente durante todo el periodo de análisis. Por otro lado, el Índice de Dependencia creció de $47 \%$ en el año 2000 a $65 \%$ en el año 2015. Si bien en el periodo previo al APC en ID subió hasta 54\%, lo que constituye un incremento de $7 \%$ en nueve años. Es desde el año 2009 que se incrementa hasta $65 \%$ en año 2015 , significando un crecimiento de $1 \%$ en 6 años (Tabla 5).

Sin embargo, el análisis del ID nos muestra la participación relativas de las importaciones. Pero si a esto le sumamos que la demanda en conjunto creció, como se aprecia en la Tabla 5. El crecimiento de las importaciones es aún mayor si lo analizamos por separado. Se puede apreciar en la Tabla 6 que el crecimiento acumulado de 
las importaciones, comparado con el año 2000, llegó al año 2016 con un acumulado de $256 \%$, que es su real crecimiento.

Tabla 5. Demanda por MAD en el Perú e Índice de Independencia

(en Ton.)

\begin{tabular}{rrrrr}
\hline Año & Importación & Producción & Demanda & ID \\
\hline 2000 & 846424 & 960362 & 1806786 & 47 \\
2001 & 843970 & 1057355 & 1901325 & 44 \\
2002 & 927089 & 1038117 & 1965206 & 47 \\
2003 & 966644 & 1097337 & 2063981 & 47 \\
2004 & 1086892 & 983156 & 2070048 & 53 \\
2005 & 1300386 & 999274 & 2299660 & 57 \\
2006 & 1502501 & 1020042 & 2522543 & 60 \\
2007 & 1523121 & 1122918 & 2646039 & 58 \\
2008 & 1391379 & 1231516 & 2622895 & 53 \\
2009 & 1499244 & 1273943 & 2773187 & 54 \\
2010 & 1903272 & 1283621 & 3186893 & 60 \\
2011 & 1897582 & 1260123 & 3157705 & 60 \\
2012 & 1831245 & 1392972 & 3224217 & 57 \\
2013 & 2005137 & 1365239 & 3370376 & 59 \\
2014 & 2315888 & 1227562 & 3543450 & 65 \\
2015 & 2661281 & 1438562 & 4099843 & 65 \\
2016 & 3020775 & & & \\
\hline
\end{tabular}

Para estimar los determinantes de la demanda se utilizó la data que se presenta en la Tabla 7. Se utilizó el precio en chacra al no haber una data completa del precio a nivel mayorista del MAD. La demanda se tomó de la Tabla 5. La variable dummy aplica el valor de "1" desde el año 2009, cuando entra en vigencia en ACP Perú - EE.UU.

Tabla 6. Crecimiento de las importaciones de MAD al

\begin{tabular}{cccc}
\multicolumn{4}{c}{ Perú } \\
\hline Año & $\begin{array}{c}\text { Importación } \\
\text { (en Ton.) }\end{array}$ & $\begin{array}{c}\text { Anual } \\
(\%)\end{array}$ & $\begin{array}{c}\text { Acumulado } \\
(\%)\end{array}$ \\
\hline 2000 & 846424 & & \\
2001 & 843970 & $-0,3$ & -0, \\
2002 & 927089 & 9,8 & 9,5 \\
2003 & 966644 & 4,3 & 14,2 \\
2004 & 1086892 & 12,4 & 28,4 \\
2005 & 1300386 & 19,6 & 53,6 \\
2006 & 1502501 & 15,5 & 77,5 \\
2007 & 1523121 & 1,4 & 79,9 \\
2008 & 1391379 & $-8,6$ & 64,4 \\
2009 & 1499244 & 7,8 & 77,1 \\
2010 & 1903272 & 26,9 & 124,9 \\
2011 & 1897582 & $-0,3$ & 124,2 \\
2012 & 1831245 & $-3,5$ & 116,4 \\
2013 & 2005137 & 9,5 & 136,9 \\
2014 & 2315888 & 15,5 & 173,6 \\
2015 & 2661281 & 14,9 & 214,4 \\
2016 & 3020775 & 13,5 & 256,9 \\
\hline
\end{tabular}

Tabla 7. Datos para evaluar los determinantes de la demanda

\begin{tabular}{cccccr}
\hline & $\begin{array}{c}\text { Demanda } \\
\text { (Ton.) }\end{array}$ & $\begin{array}{c}\text { Precio } \\
\text { Chacra } \\
\text { S/./ Kg) }\end{array}$ & $\begin{array}{c}\text { PBI } \\
\text { (Soles) }\end{array}$ & $\begin{array}{c}\text { Pob. } \\
(\text { Hab. })\end{array}$ & DAPC \\
\hline 2000 & 1806,786 & 0,51 & 222,207 & $25,939,329$ & 0 \\
2001 & 1901,325 & 0,51 & 223,580 & $26,328,938$ & 0 \\
2002 & 1965,206 & 0,51 & 235,773 & $26,724,398$ & 0 \\
2003 & 2063,981 & 0,49 & 245,593 & $27,125,799$ & 0 \\
2004 & 2070,048 & 0,54 & 257,770 & $27,533,228$ & 0 \\
2005 & 2299,660 & 0,49 & 273,971 & $27,946,774$ & 0 \\
2006 & 2522,543 & 0,53 & 294,598 & $28,337,749$ & 0 \\
2007 & 2646,039 & 0,66 & 319,693 & $28,734,194$ & 0 \\
2008 & 2622,895 & 0,74 & 348,923 & $29,136,186$ & 0 \\
2009 & 2773,187 & 0,68 & 352,584 & $29,543,801$ & 1 \\
2010 & 3186,893 & 0,75 & 382,380 & $29,957,804$ & 1 \\
2011 & 3157,705 & 0,91 & 407,052 & $30,350,251$ & 1 \\
2012 & 3224,217 & 0,83 & 431,273 & $30,747,840$ & 1 \\
2013 & 3370,376 & 0,88 & 456,366 & $31,150,636$ & 1 \\
2014 & 3543,450 & 0,90 & 467,280 & $31,558,710$ & 1 \\
2015 & 4099,843 & 0,90 & 482,797 & $31,972,027$ & 1 \\
2016 & & & 501,699 & $32,353,134$ & 1 \\
\hline Fuente: MINAG (2006). & & & &
\end{tabular}

Al evaluar las variables en modelos de regresión se encontró que las variables Precio en Chacra y la variable Dummy no eran significativas para el modelo. Con lo que la demanda se explicaría por la influencia del PBI y del crecimiento poblacional. La forma final del modelo fue en base a los logaritmos de las variables mencionadas:

El modelo sería entonces:

Ldemanda $=\mathrm{c}(1)+\mathrm{c}(3) * \mathrm{Lpbi}+\mathrm{c}(4) * \mathrm{Lpob}$

Donde: Ldemanda $=$ Logaritmo de la demanda

Lpbi $=$ Logaritmo del PBI

Lpob $=$ Logaritmo de la población

Así, los coeficientes asociados a las variables explicativas vendrían a ser elasticidades:

Elasticidad PBI $=0,062$

Lo que nos dice que un incremento en $1 \%$ en el PBI genera Elasticidad Población $=3,45$

Nos dice que un incremento en $1 \%$ en la población genera un incremento de $3,45 \%$ en la demanda de MAD.

Habría que considerar que la demanda por MAD por parte de la población es una demanda indirecta pues la población lo consume a través de los productos para los que se utiliza el MAD, que son la carne de ave, de porcino y de vacuno.

Tabla 8. Reporte de la regresión que explica la demanda 
de MAD

Dependent Variable: LDEMANDA

Method: Least Squares

Date: 10/25/17 Time: 11:14

Sample(adjusted): 20002015

Included observations: 16 after adjusting endpoints

LDEMANDA $=\mathrm{C}(1)+\mathrm{C}(3) * \mathrm{LPBI}+\mathrm{C}(4) * \mathrm{LPOB}$

\begin{tabular}{lrlrr}
\hline & Coefficient & Std. Error & t-Statistic & Prob. \\
\multicolumn{1}{c}{ C $(1)$} & -45.34233 & 21.30010 & -2.128737 & 0.0530 \\
C(3) & 0.062749 & 0.364255 & 0.172266 & 0.8659 \\
C (4) & 3.453491 & 1.507481 & 2.290903 & 0.0393 \\
R-squared & 0.979513 & Mean dependent var & 14.78101 \\
Adjusted R-squared & 0.976361 & S.D. dependent var & 0.248873 \\
S.E. of regression & 0.038264 & Akaike info criterion & -3.521251 \\
Sum squared resid & 0.019034 & Schwarz criterion & -3.376391 \\
Log likelihood & 31.17001 & F-statistic & 310.7735 \\
Durbin-Watson stat & 1.708811 & Prob(F-statistic) & 0.000000 \\
\hline
\end{tabular}

\section{Conclusiones}

El APC Perú - EE.UU no hizo que la producción de MAD disminuya en Perú, como un estudio había pronosticado. Si no que por el contrario creció aún son contar con el subsidio, que era la forma en que el Estudio de IICA sostenía que se iba a realizar. El incremento de las importaciones desde Estados Unidos creció a un ritmo mayor a la cuota de importación establecida en el APC. Aunque el Perú era un país con dependencia de MAD importado y este índice venía creciendo. El APC con EE.UU. hizo que el crecimiento de este índice de dependencia se acelere.

Con lo que el Perú es un país más dependiente de MAD importado, en especial de Estados Unidos. Finalmente, La demanda por MAD se estima por el PBI y por la población.

Así el crecimiento económico registrado en el Perú durante el periodo de análisis hizo que la demanda por MAD se incremente. También el crecimiento de la población hizo que la demanda por MAD se incremente al incidir de manera directa en el consumo de carnes que tiene como insumo el MAD.

\section{Literatura citada}

Cafferata, J. y Arias J. 2007. El acuerdo de promoción comercial Perú-EE.UU. Posibles impactos en la agricultura peruana. Comuniica (Año 3 Segunda etapa, Mayo - Agosto 2007). Disponible en: http://repiica. iica.int/docs/B0577e/B0577e.pdf.

CEPES-CONVEAGRO. 2009. Impacto Del TLC Perú-EE. UU. En el maíz amarillo duro - Preliminar. Disponible en: http://www.cepes.org.pe/apc-aa/archivos-aa/a01e3 bc3e44a89cf3cd03d717396a20e/Impacto_TLC_Maiz_ Amarillo_Duro.pdf

De Ita, A. 2008. Disponible en: http://www.bilaterals.org/ IMG/pdf/Catorce_anos_del_TLCAN_y_la_crisis_de_ la_tortilla.pdf

FAO. 2010. Anuario estadístico de la FAO 2014. Disponible en: www.fao.org/3/a-i3592s.pdf

MINAG. 2006. Informe Técnico Resultados Del Tratado de Libre Comercio Perú -Estados Unidos, Sector Agricultura. Disponible en: http://www.inbior.com/ documentos/Agro-TLC.pdf

Moreno, L.; Gonzáles, S. y Matus J. 2016. Dependencia de México a las importaciones de maíz en la era del TLCAN. Revista Mexicana de Ciencias Agrícolas, 7(1): 115-126. 